\title{
The Various Stress Patterns of Press-Fit, Ingrown, and Cemented Femoral Stems
}

\author{
R. HUISKES, PH.D.
}

Finite-element analysis was used to study the general differences in load-transfer mechanisms and stress patterns of cemented, fully ingrown, proximally ingrown, and smooth press-fitted femoral stems in total hip arthroplasty (THA). Identical stems were used for the noncemented configurations and a similar stem shape for the cemented configurations. In each model, bone properties and loading characteristics were equal. Stem elastic moduli were varied so that the effects of cobaltchromium-molybdenum (CoCrMo) and titanium as different stem materials could be assessed. The load-transfer mechanism is similar for all bonded configurations but differs dramatically for unbonded stems, e.g., press-fit designs. In the bonded configurations, interface stress concentrations occur on the proximal and distal sides. Stress value depends on stem rigidity, with higher proximal stress occurring in cemented stems and higher distal stress in noncemented stems. In the press-fit stem, the interface stresses are affected more by stem shape as a geometric entity and less by stem rigidity. Considering possible postoperative failure mechanisms, such as interface loosening and cortical bone loss, titanium is expected to produce better results in noncemented stems and CoCrMo in cemented stems. Cortical stress shielding as a qualitative phenomenon is caused by all stems, particularly in the calcar region. Quantitatively, stress-shielding effects differ with each type of fixation used. Stress-shielding effects are severe in fully ingrown stems and milder in cemented stems because of the differences in stem rigidity. The

Sponsored in part by Osteonics. Allendale. New Jersey.

Presented at the 18 thin Open Scientific Meeting of the Hip Society, New Orleans, Louisiana, February 11. 1990.

Reprint requests to R. Huiskes, Ph.D., Biomechanics Section, Institute of Orthopaesdics. University of Nijmegen, 6500 HB Nijmegen, the Netherlands.

Received: April 4, 1990. proximally ingrown stem falls between the fully ingrown and cemented stems in regard to stress shielding because stress transfer is more evenly distributed along the stem and concentrated at the lower coated edge. The press-fit stem provokes calcar stress shielding only. In the midstem region the stresses in the cortex are even greater than in the natural case.

The transfer of load from prosthesis to bone is one of the most important functions of a femoral hip stem. The stress patterns associated with the load-transfer mechanism may have important effects on the endurance of the prosthesis. Long-term follow-up studies of cemented total hip prostheses have indicated that prosthetic loosening is frequently associated with cement (fatigue) fracture or failure of the cement-bone and stem-cement interfaces or both. ${ }^{8.20,27}$ Noncemented stems lack the relative mechanical weaknesses of acrylic cement and its interfaces with metal and bone. However, excessive stem-bone interface stresses may cause local relative (micro) motions that could provoke midthigh pain, prevent bony ingrowth and osseus integration, or produce interface bone resorption and fibrous-tissue interposition..$^{8,10,30}$ Noncemented stems are known to produce proximal bone resorption because of strain-adaptive bone remodeling caused by excessive stress shielding., ${ }^{9,17,18,28}$ This effect also threatens the endurance of the system and is related to the stress patterns associated with the load transfer mechanism.

It is important for clinicians and prosthetic designers to develop an understanding of the 
bone-prosthesis structure, and the side plate accounts for the three-dimensional structural integrity of the bone. The element thicknesses were nonuniform and determined from the real thicknesses of the separate components. In this way, the flexural rigidities of the stem and the bone in the midfrontal plane were reproduced in the model. ${ }^{13,16,29}$ Characteristic elastic moduli were taken for the stem (cobalt-chromium-molybdenum [CoCrMo], $2.0 \times 10^{5} \mathrm{MPa}$; titanium, 1.1 $\left.\times 10^{5} \mathrm{MPa}\right)$, for the cortical bone front and side plates $\left(1.7 \times 10^{4} \mathrm{MPa}\right)$, for the metaphyseal cortex $\left(0.5 \times 10^{4} \mathrm{MPa}\right)$, for the trabecular bone $\left(1.0 \times 10^{3}\right.$ $\mathrm{MPa})$, and for the acrylic cement $\left(2.0 \times 10^{3} \mathrm{MPa}\right)$. Elements were plane-stress quadrilaterals with four nodal points each.

Three loading cases were considered (Fig. 1): $F_{1}$ according to the one-legged stance in gait, ${ }^{6} \mathrm{~F}_{2}$ lateralized $10^{\circ}$, and a pure bending moment, $M$. Force values up to $3000 \mathrm{~N}$ were considered, about four times body weight.

The four configurations analyzed are shown in Figure 2; cemented, fully coated cementless, partly coated, and press-fit stems. All stem shapes were taken from Osteonics Omnifit prostheses (Allendale, New Jersey), Size 7 for the cemented one, and Size 10 for the noncemented ones. The cemented and fully coated configurations had bonded interfaces, allowing compressive, tensile, and shear stresses to be transferred. The cemented stem was assumed loose from the cement mantle along the upper proximolateral $10-\mathrm{mm}$ distance. The tip of the noncemented stem was not in contact with any bone. The partly coated stem was assumed fully bonded at the coated sites only. The distal part of the partly coated stem and the pressfit stem over its full length were assumed loose,
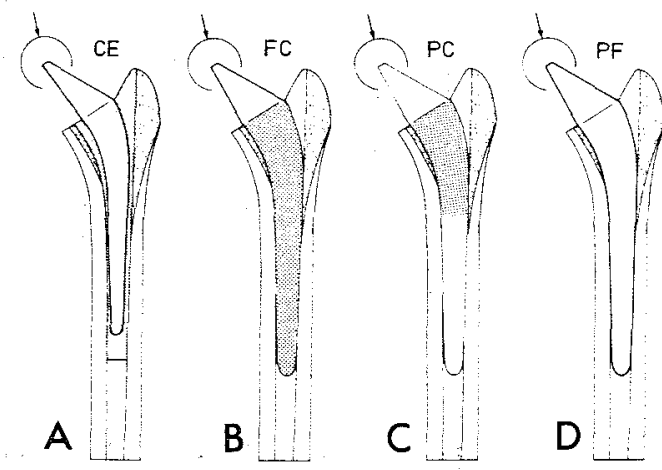

FIGs. 2A-2D. The four stem configurations analyzed in the study: (A) cemented (CE), (B) fully ingrown (fully coated, $\mathrm{FC}$ ), $(\mathrm{C})$ partly ingrown (partly coated, PC), and (D) smooth press fitted (PF). allowing compressive stress transfer only. Slip and tensile separation may occur, owing to the nonlinear gap elements applied (Marc Analysis, Palo Alto, California). The solution was obtained iteratively in this case of loose interfaces. In all four cases, the bone geometry and loading conditions were identical.

The stems were assumed to be made out of CoCrMo, but the effects of titanium were studied as well by reducing the elastic moduli of the stems. In addition to the above models, a finite-element analysis was carried out on the same bone without a prosthesis, as in the natural case, for the same loads.

\section{RESULTS}

The stress patterns obtained in the cemented model were typical for the loadtransfer mechanism of cemented prostheses in general for loading case $F_{1}$ (Fig. 3). The predominant effect of the load was bending, generating compressive stresses on the medial surfaces, and tensile stresses on the lateral periosteal surfaces of the bone in the longitudinal direction (bending stresses).

The stem was also loaded predominantly in bending, with longitudinal compression along its medial faces and tension along its lateral faces (Fig. 3). The medial compressive stress values increased from about $80 \mathrm{MPa}$ on the proximomedial side to a maximum of about $140 \mathrm{MPa}$ at one-third of the stem length, then gradually reduced to zero $\mathrm{MPa}$ at the tip. The tensile bending stress pattern on the lateral side was similar, with a maximum of about $100 \mathrm{MPa}$, which was lower than the compression on the medial side.

As is typical for intramedullary stem fixation, concentrations of stress transfer between stem and bone occur proximally and distally. This has consequences for the stresses at the stem-cement and the cementbone interfaces and in the cement itself in these regions. Interface stress transfer can be characterized by shear and normal (perpendicular) stress components, the latter of which is either tensile or compressive. The normal and shear stress patterns at the medial and lateral stem-cement interfaces are also shown in Figure 3. The proximal stress 
load-transfer mechanism and its associated stress patterns in total hip prostheses, its relationships with the particular mechanical characteristics of the prosthesis, and the chances for its long-term survival. The finiteelement method is preeminently suited to provide information in this respect. ${ }^{16}$ The stress patterns that occur in cemented femoral prostheses have been documented using this method. 2,4,5,13-15,22,25.26. The principles of the load-transfer mechanism have been evaluated and the effects of load, stem shape, material property, acrylic cement thickness, and calcar-collar contact have been studied. Generally, the analyses have rendered an awareness of biomechanical stress phenomena. Finite-element analyses of noncemented stems have been reported much less frequently. ${ }^{17,24}$

The finite-element studies of cemented prostheses have shown that the load-transfer mechanism in femoral stems, although relatively simple in principle, ${ }^{13}$ creates complex stress patterns in the separate materials involved and at their interfaces. The stress patterns depend on the particular loading characteristics, the shape of the stem, and the surrounding structures such as acrylic cement, trabecular and cortical bone, the elastic properties of these materials, and the bonding characteristics of the interfaces. Inevitably, a high variability in results has been reported in both analytic and experimental investigations, depending on the characteristics of the particular system studied. This emphasizes the need for generalized information about the fundamental relationships between the stress patterns and the above-mentioned characteristics.

The purpose of this study was to investigate differences between stress patterns as occurring in cemented, ingrown (or osseous integrated), and press-fitted femoral prostheses. The objective was not to produce detailed information on stress values occurring in particular types of prostheses but rather to evaluate the principal, general effects of these three methods of fixation for the load-transfer mechanism.
For this purpose, a generalized finite-element model was developed in which the loading characteristics, the bone geometry, and the elastic properties for each fixation method were equal. The stem thickness differed only for the cemented case but was still similar in shape.

\section{MATERIALS AND METHODS}

Two-dimensional side-plate models were used for the analyses. ${ }^{13}$ The frontplate of such a model (Fig. 1) reproduces the midfrontal plane of the

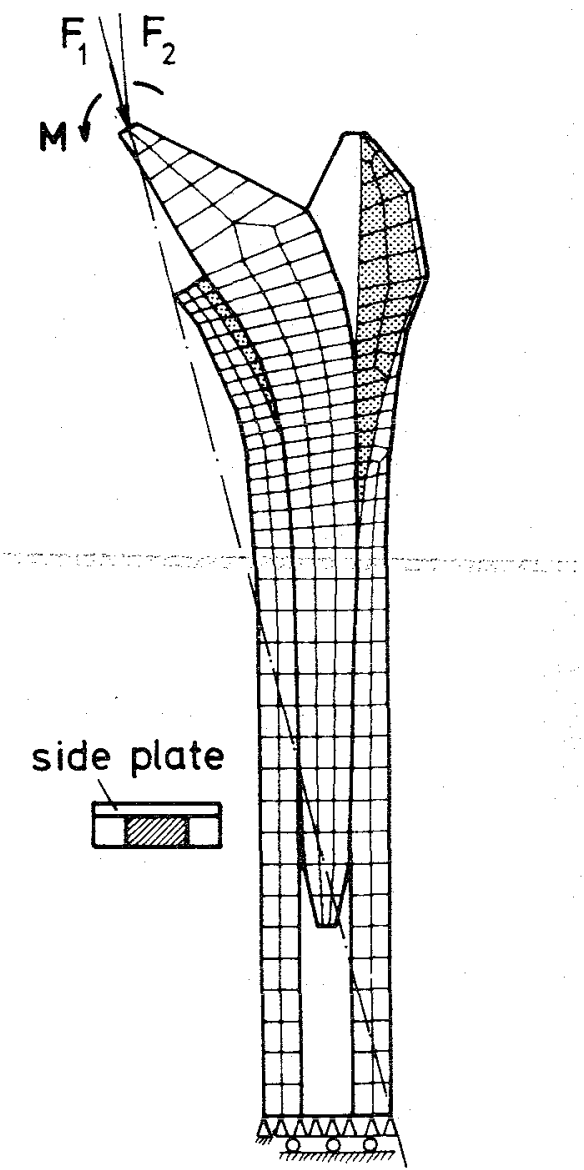

FIG. 1. Finite-element model of the prosthetic configuration of a noncemented stem. The front plate is shown. Three-dimensional bone integrity and rigidity characteristics in the frontal plane are accounted for by a side plate and nonuniform element thicknesses. Three hip-joint-loading cases are indicated, pure bending and two forces $(3000$ N). $F_{2}$ is rotated $10^{\circ}$ laterally relative to $F_{1}$. 

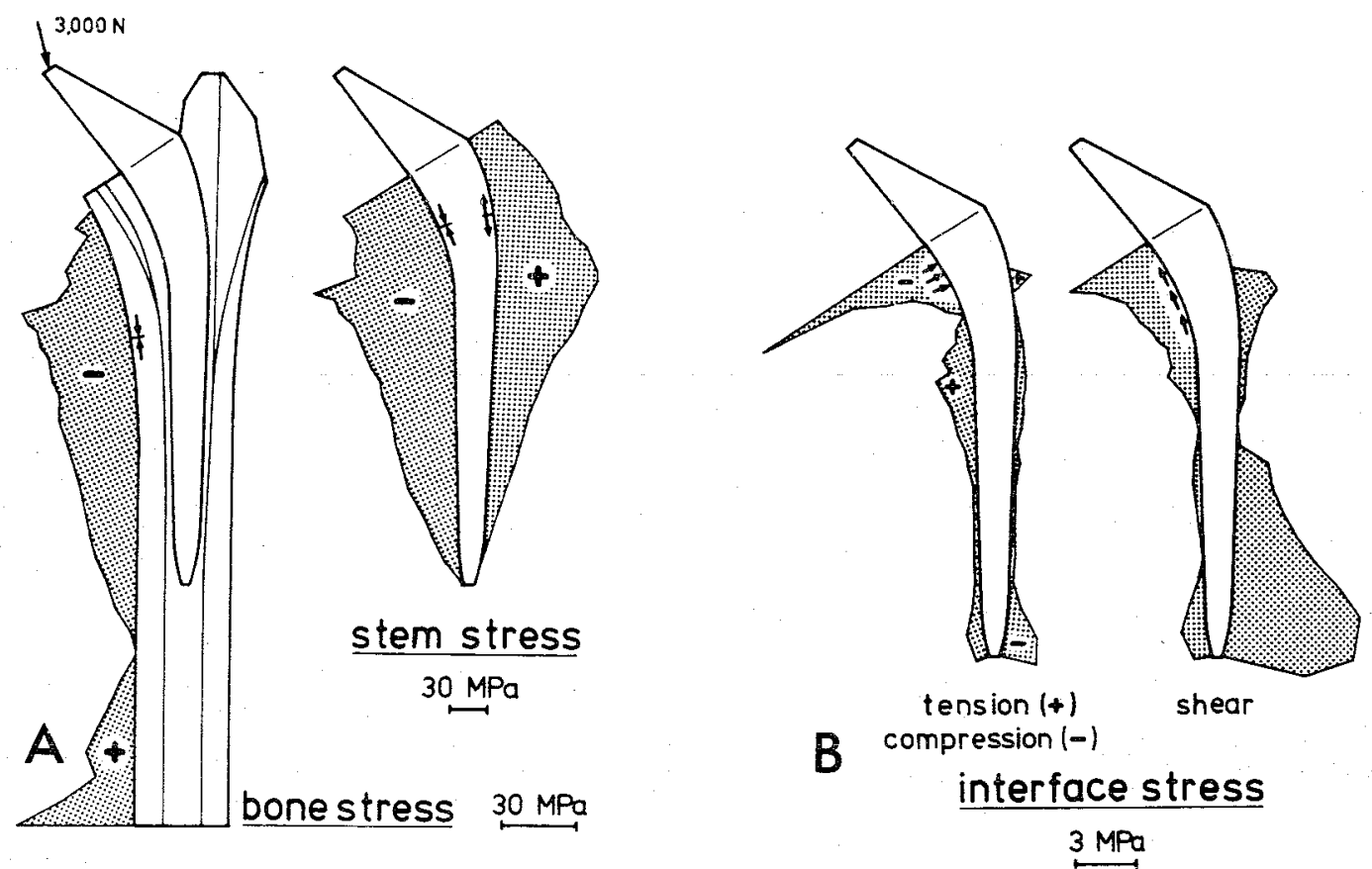

FIGS. 3A AND 3B. (A) Bending stresses at the medial periosteal bone surface and the medial and lateral faces of the stem as found for loading case $F_{1}$ in the cemented stem model. Positive represents tensile and negative represents compressive stress. (B) Normal, direct stresses (tension, compression) and shear stresses at the medial and lateral stem-cement interfaces for the sane case:

concentrations are high compared to the distal ones, owing to the strongly tapered shape of the stem. These stresses are directly transferred to the bone through the cement layer. Hence, similar patterns are found in the cement and along the cement-bone interface.

When the external force is rotated laterally by $10^{\circ}$ as in loading case $\mathrm{F}_{2}$, its bending effect increases. As a result, almost all stress values increase, although the general patterns remain similar. The maximal medial compressive stress in the stem due to bending increases from 80 to $190 \mathrm{MPa}$, and the maximal lateral tensile stress increases from 140 to $155 \mathrm{MPa}$. Proximal interface and cement stresses increased by about $50 \%$. Most distal stresses increase by factors of two to four, but the maximal distolateral shear stress reduces by about $60 \%$.

The stress patterns for the noncemented, fully coated (and ingrown) stems followed the same general principles as the cemented one. The differences were mainly due to the increased rigidity of the thick, canal-filling stem. The stem was again loaded predominantly in bending. Its bending stress values were about $30 \%$ less than in the cemented case, about $120 \mathrm{MPa}$ maximal medial compression and $110 \mathrm{MPa}$ maximal lateral tension for the lateralized force $F_{2}$. These maximal values occurred in the midstem region, which was more distal when compared to the cemented stem.

The interface stress patterns with concentrations on the proximal and distal sides were also similar to the cemented case, but the values shifted, with a reduction of about $30 \%$ on the proximal side and up to four times higher on the distal side. Hence, relative to the cemented prosthesis, more load was transferred distally and less proximally, which was purely an effect of the relative rigidity of the noncemented stem (Fig. 4).

The shift in load transfer also affects the stresses in the cortex, as illustrated in Figure 5 , showing medial endosteal bending stresses 

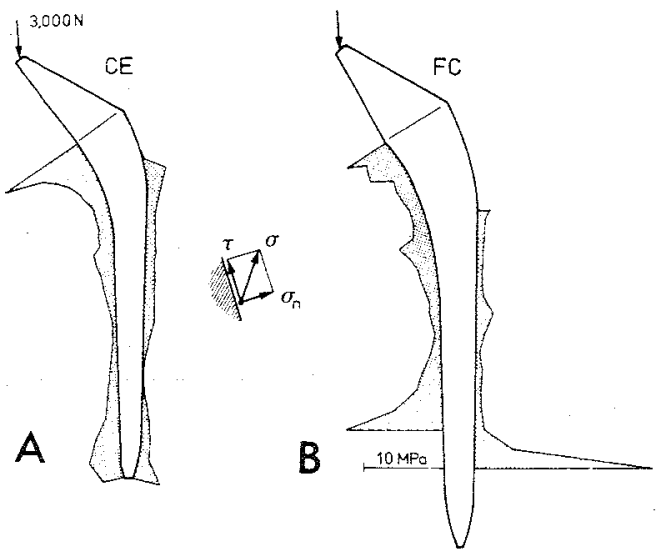

FIGS. 4A AND 4B. Interface stresses at the (A) cemented and (B) fully coated medial and lateral stem interfaces (loading case $F_{2}$ ). Shown is the stress $\sigma$, which is the vector sum of the normal direct interface stress $\sigma_{\mathrm{n}}$ and the interface shear stress $\tau\left(\sigma=\left(\sigma_{\mathrm{n}}^{2}+\tau^{2}\right)^{1 / 2}\right)$. CE, cemented; FC, fully coated.

as percentages of the natural ones for the cemented and fully coated stems. At the calcar, both stems caused similar stress-shielding effects. Farther distally, however, the stressshielding effects of the bulky, noncemented stem were much more severe than those of the relatively flexible cemented one.

If the stems were made of titanium instead of CoCrMo alloy, their rigidities were reduced by almost $50 \%$. The principles of intramedullary load transfer dictate ${ }^{13,22}$ that stem loading decrease (the stem takes a smaller portion of the load and the bone more). Hence, the bone stresses increase and more

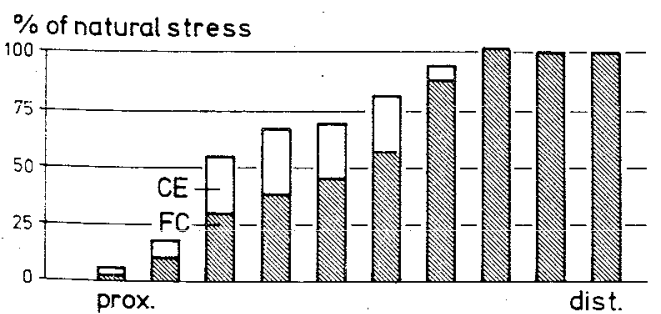

FIG. 5. Bone stresses along the medial periosteal cortex for the cemented (CE) and fully coated (FC) stems (both CoCrMo) as percentages of stresses occurring in the natural bone, illustrating the stress-shielding mechanism (loading case $\mathbf{M}$ ). prox., proximal; dist.. distal.

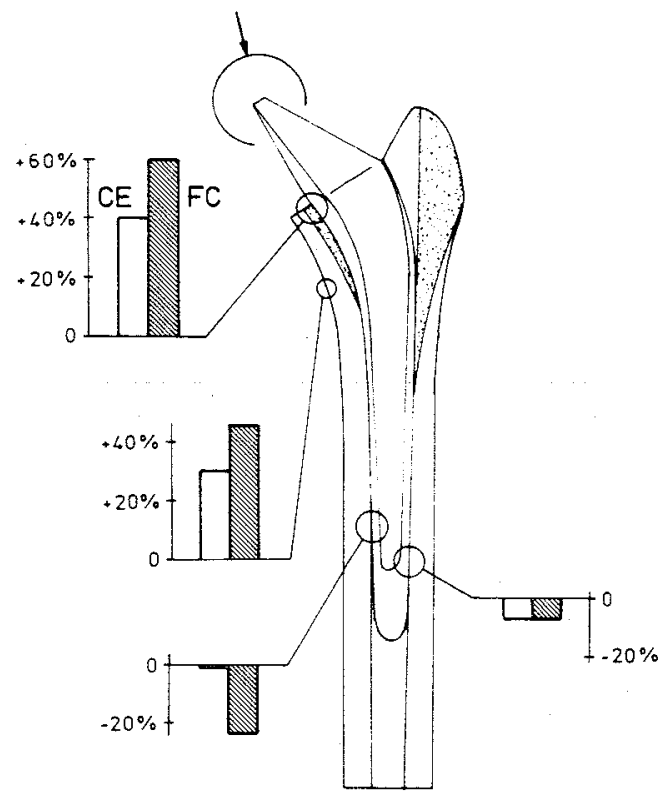

FIG. 6. Percentile effects of titanium versus CoCrMo alloy as stem material on the proximal and distal interface stresses and on the periosteal cortex stress for the cemented (CE) and fully coated (FC) stems (loading case $F_{1}$ ).

load is transferred proximally and less distally. These principles are reproduced in the present results (Fig. 6). The effects were more pronounced for the noncemented stem than for the cemented one. Proximal interface stresses (and also cement stresses in the cemented configuration) increased by $40 \%$ and $60 \%$, respectively. Distal interface (and cement) stresses were reduced by $1 \%$ to $8 \%$ and $8 \%$ to $21 \%$, respectively. Stresses in the medial cortex increased by about $30 \%$ in the cemented configurations and by about $46 \%$ in the noncemented configurations.

Compared to the fully coated stem, the partly coated stem transferred more stress in the proximal part and less in the distal uncoated part. Stress transfer in the proximal methaphysis was very similar, but stress concentrations occurred near the lower end of the coating, particularly in shear stress. The most important effects on the interface stresses are summarized in Figure 7. Relative to the fully coated stem case, the calcar interface stress remained unchanged. Near the 


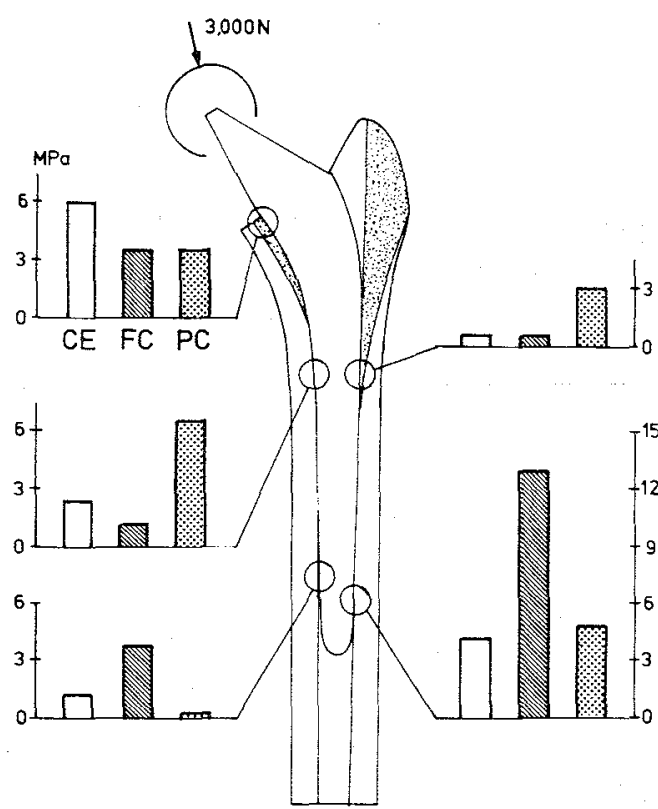

FIG. 7. Interface stresses at the proximal, midstem, and distal regions for the cemented (CE), fully coated (FC), and partly coated (PC) configurations (loading ease $F_{1}$ ). The interface stresses are again the resultants $\sigma$ of the interface normal $\left(\sigma_{n}\right)$ and shear $(\tau)$ stresses (compare Figure 4 ).

distal coating edge, in the midstem region, medial and lateral interface stresses increased by factors of more than five. At the distal tip of the stem, the stresses at the loose interface reduced by factors of three (lateral side) and ten (medial side).

As a consequence of this shift in load transfer from the distal tip to the coating edge, the stress-shielding effects in the cortex were much less severe in the midstem region (Fig. 8). In the calcar region, the cortical stresses were very similar in value. Farther distally, however, the stress-shielding effects around the partly coated stem were reduced relative to the fully coated stem. From the midstem region, cortical stress values around the partly coated stem were very similar to those around the cemented one.

The stress-transfer mechanism for the press-fit stem was dramatically different from the three other cases because the interface bond was uncoupled and the stem could sub- side elastically when loaded. Interface stress transfer must rely exclusively on compression since tension cannot exist, and, in the absence of friction, nor can shear stress. Because the taper angle of the stem is relatively small, high compressive interface stress must be developed to equilibrate the axial force component. In the other three cases, in which the stem was bonded, equilibrium was maintained predominantly by the interface shear stresses. Consequently, the interface (compressive) stresses for the press-fit stem are three to seven times higher than those for the partly coated stem (Fig. 9). Stresses are no longer concentrated in the proximal and dis-

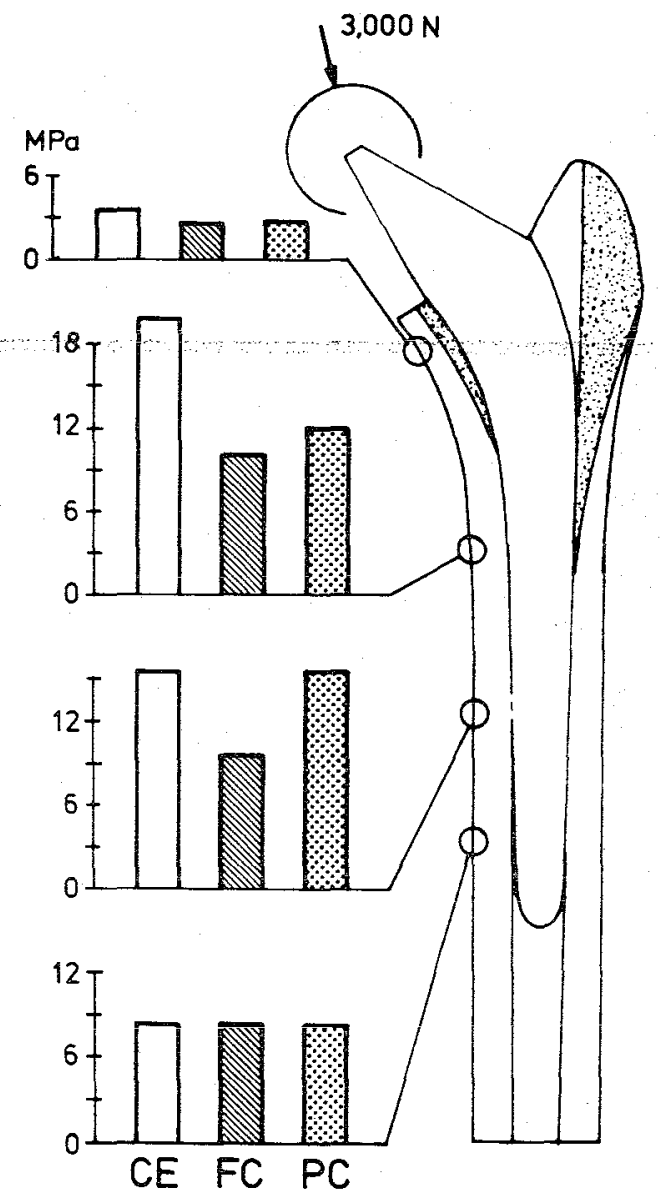

FIG. 8. Bone stresses at four locations along the medial periosteal cortex for the cemented (CE), fully coated (FC). and partly coated (PC) stems (loading case $F_{1}$ ). 


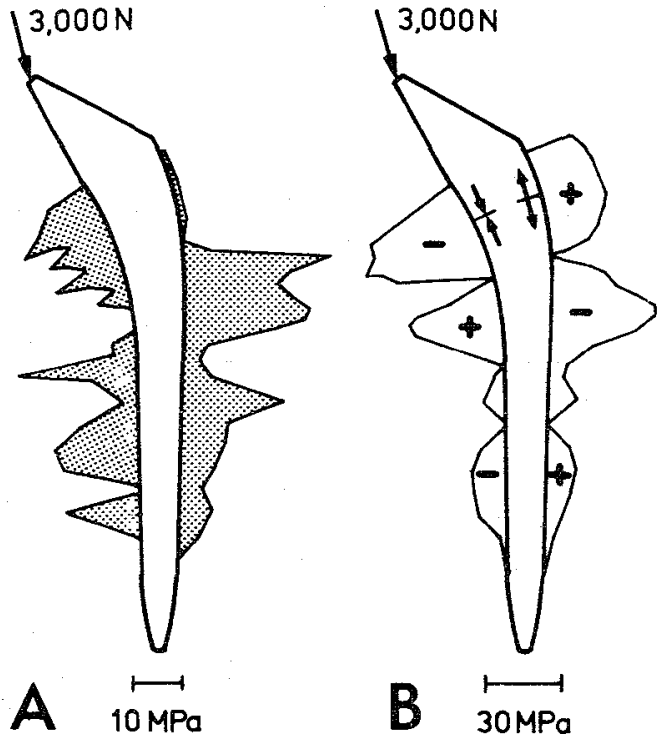

FIGs. 9A AND 9B. The press-fitted stem (loadirig case $F_{1}$ ). (A) Compressive interface stresses. (B) Stem bending stresses. Positive represents tension and negative represents compression.

tal areas. Notable is the compression that occurs in the lateral upper midstem region, just below the lateral curve of the stem (Fig. 9). This is a combined effect of elastic subsidence and stem curvature. Because of its curvature, subsidence of the proximal stem is resisted by the calcar and the stem is pushed laterally against the endosteal bone. This mechanism causes a peculiar bending pattern of the stem, also shown in Figure 9. For the bonded stem cases, (compare Fig. 3), cantilever beam bending in the stem was noticed because of evidence of compression at the medial faces and tension at the lateral faces. in the press-fit case, however, the stem was bent medially in the proximal and lateral areas and laterally in the midregion, similar to a stem subjected to a four-point bending load.

The cortical bone stresses around the press-fit stem were also higher than those around the bonded stem, although some stress shielding still occurred in the calcar region. Halfway along the stem, the cortical bone stresses were about three times as high as those for the cemented stem case and about twice as high as the intact natural bone.
The effects of the laterally rotated force $F_{2}$ compared to the reference force $F_{1}$ on the stress values were much less significant for the press-fit stem as compared to the other three cases. The same was found when changing the stem material from CoCrMo alloy to titanium. Both these findings indicate that in the case of unbonded interfaces, the axial force component of the hip-joint load is more prominent for the load-transfer mechanism than the bending component, contrary to what was found for bonded interfaces.

\section{DISCUSSION}

The present study used a two-dimensional, finite-element side-plate model to document the differences in stress transfer around cemented, bony ingrown (osseous integrated), and smooth press-fitted hip stems of similar shapes. Cement interfaces were assumed to be well bonded, coatings were assumed fully ingrown, and press-fitted interfaces were modeled as line-to-line interference fits. As a consequence of this schematic approach the results must be interpreted relative to each other, as indicative for the load-transfer mechanisms in the different cases.

The two-dimensional side-plate model with nonuniform element thickness was used before, and comparisons with other models and experimental data have been made. ${ }^{13-15}$ In a recent study, ${ }^{29}$ it was shown that the stress patterns determined in such a model were equal to those determined in the midfrontal plane in symmetric, three-dimensional, finite-element models with symmetric loading such as those applied by Crowninshield et al. ${ }^{4}$ However, cortical hoop stress cannot be determined in the two-dimensional case, although its effects are accounted for by the side plate. Also, effects of torsion cannot be studied.

Nonsymmetric (anatomic) models are closer to geometric reality and show local stress details that are not seen in symmetric ones. ${ }^{2,15,22,25,26,29}$ However, under symmetric. frontal plane loading, the load-transfer mechanism and the general stress patterns of these 
anatomic models are reproduced in the three-dimensional symmetric and two-dimensional side-plate models. ${ }^{15,29}$ The latter model is less adequate when interfaces are unbonded.

In the analyses, three hip-joint-loading cases were investigated. No trochanteric loading was assumed. Evidently, trochanteric loading changes the stress values in all materials to a significant degree. ${ }^{11,25}$ However, the emphasis in this study was on the loadtransfer mechanism from stem to bone, which is more easily interpreted and compared when only hip-joint loading is considered. The pure bending moment considered is not a physiologic load, but for several reasons it is well-suited to compare load-transfer mechanisms and bone stresses. ${ }^{18,25}$ The present results showed that the bending component of the hip-joint force most predominantly determines the stress patterns in the system rather than the axial (compressive) force atone, as long as the interfaces are bonded.

The differences in load-transfer between cemented and fully coated (fully ingrown) stems are quantitative, not qualitative, in nature. The general patterns of cortical stress shielding, predominantly proximal and distal load transfer, and stem bending patterns are reproduced in both cases. Quantitative differences are due to the relative rigidities of the stems. The flexural rigidity of the noncemented stem is about 3.6 times as high as the cemented one (midstem region). As a result, distal interface stress transfer increases at the expense of proximal stress transfer, and stress-shielding effects in the cortex area increase. These general rules for the effects of stem rigidity were established earlier. 4,13,22

If the stems were made of titanium, their rigidities would be reduced by almost $50 \%$; hence, proximal stress transfer would be increased over distal stress transfer. Because of the relatively high proximal interface stresses created by the cemented stem and the relative weakness of these interfaces, ${ }^{1,21}$ changing to titanium would have been a poor alternative. Conversely, ingrown or osseous-integrated interfaces are much stronger, ${ }^{3,12}$ and the distal interface stresses are higher than the proximal ones in the ingrown case. Hence, changing the noncemented ingrown stem to titanium would have been a valid option, and, in view of reduced stress shielding, a good alternative. The noncemented stem made of titanium would still be almost twice as rigid as the cemented one made of CoCrMo alloy.

Changing from a fully coated to a partly coated configuration shifts the distal shear stress concentration to the midstem region (near the edge of the coating) without increasing its maximal values. This phenomenon seems advantageous because it tends to involve the midstem part in stress transfer. Relative to the fully ingrown case, the stress transfer becomes more uniformly distributed over the stem length. Clinical results of prostheses proximally coated with hydroxyapatite show that bone remodeling (roentgenographic densification) occurs precisely where the interface stress concentrations occur, near the distal coating edge. ${ }^{12}$ As a result of midstem load transfer, the stress-shielding effects in the cortex are also reduced relative to the fully coated configuration, reaching the same level as in the cemented case about halfway down the stem. On the proximal side, however, stress shielding is still severe in both cases, as was also found in strain-gauge analyses of other noncemented stems. ${ }^{7,11}$

The question arises as to the mechanical function of the distal, uncoated part of the stem. The bending component of the hipjoint force tends to produce distal toggling of the stem, which is resisted by compression at the laterodistal interface. It may be argued that bending is resisted by the distal stem by lateral compressive interface stress. This stress would then be lower when the stem is longer, increasing the moment arm of the interface force. It may also be argued that the tendency for distal toggling is greater when the stem is longer, because it would be farther away from the rotation center. The compressive stresses, therefore, would be lower for a longer stem. In fact, the distal interface stress depends on the length and flexural rigidity of 
the distal stem, the effect of rigidity becoming more significant when the stem is longer ${ }^{13}$ It is likely that the stem of a proximally coated prosthesis could be shortened considerably without notable effects on the load-transfer mechanism in general and with reduction of the distal stress values. An optimal value for the stem length could be determined, given the particular stem shape.

When the interfaces are completely loose, as for a smooth press-fitted stem, the load transfer mechanism changes considerably. This is purely a consequence of the opportunity for elastic subsidence (slip) of the stem within the bone. This has two distinct effects. The first is illustrated in Figure 10. When the interface is unbonded and frictionless, shear stresses can no longer be developed. The stem will then subside until the interface compression becomes high enough for its axial component to balance the axial force. The smaller the taper angle, the more compression is needed to develop the required equilibrium between the axial compressive stress component and the axial force. The smaller the taper angle, the more elastic subsidence is re-

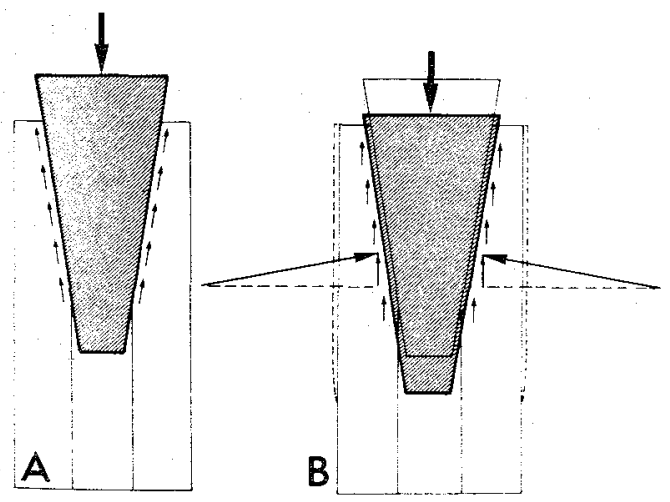

Figs. 10A AND 10B. Schematic representation of load transfer from a straight tapered cone pushed into a cylindrical counterpart. (A) With bonded interfaces, shear stress is developed to effectively equilibrate the compressive force applied. (B) At a smooth, press-fitted interface, equilibrium relies on the relatively small vertical component of compressive interface stress. The cone must subside to develop the required compressive stress values depending on the rigidity of the surrounding structure. quired. The amount of subsidence required also depends on the rigidity of the bone constraint. The stiffer the bony shell, the more compressive stress will be developed for a particular amount of subsidence. In reality, the rigidity of the bony constraint is nonuniform, i.e., much higher in the diaphysis than in the metaphysis. Therefore, the compressive stress patterns generated are also nonuniform.

Obviously, the mechanism described above would also be influenced by friction and the distribution of initial interface contact. Because of friction, shear stresses at the interface can be developed that assist the axial components of the compressive stresses in balancing the axial force. In the present model, friction is neglected; therefore, quantitatively the results obtained are exaggerated. Very little is known about friction between implant and bone. In view of the biologic (wet) environment, the coefficient of friction between a smooth implant and endosteal bone is probably very low.

When interface contact is concerned, the present model assumes an idealized line-toline interference fit. When only proximal fit is realized, the relative flexibility of the metaphyseal cancellous bone could allow subsidence until distal fit is obtained as well. If not, the distal stem would toggle in the medullary canal, possibly creating endosteal resorption and midthigh pain. If only distal fit is realized, the relative stiffness of the diaphyseal bony constraint would not allow much elastic subsidence. This would result in a proximal stress bypass, a mechanism similar to stress shielding, ${ }^{19}$ causing proximal cortical bone loss and interface resorption. Since so much depends on initial fit, which is difficult to realize consistently, ${ }^{23,32}$ it is likely that the clinical results of press-fitted stems will be somewhat unpredictable.

The second effect of loose interfaces allowing for elastic subsidence is illustrated in Figure 11. Because the stem is not a straight taper, elastic subsidence as described above is not entirely possible because it causes the interface fit to lose conformity. The proximal 


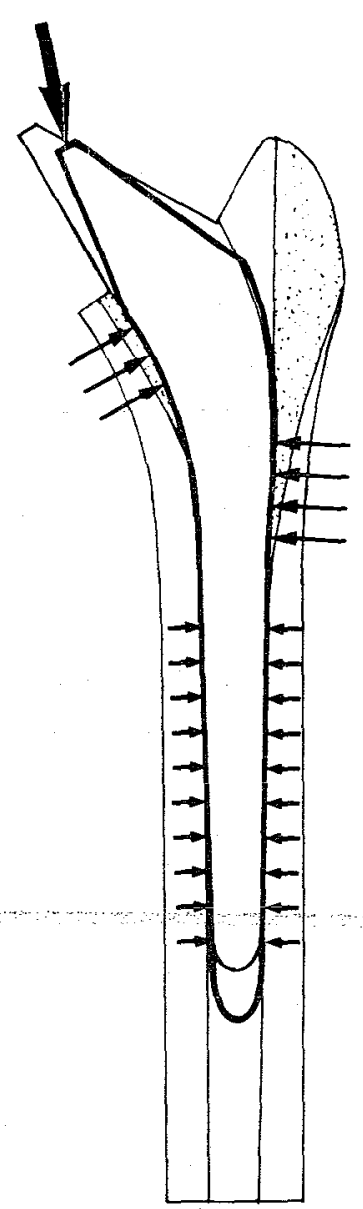

FIG. 11 . Schematic representation of the subsiding mechanism of the press-fitted stem. The distal stem acts similar to the straight tapered cone. Proximally, however, subsidence is resisted by the curvature of the stem. The surrounding bone attempts to straighten out the stem, developing high compressive stresses proximomedially and midlaterally. The amount of subsidence is greatly exaggerated to allow visualization.

curvature causes a tendency for lateral rotation of the implant, the bone constraint tending to straighten out the stem and, therefore, the peculiar bending mechanism of the stem described in the previous section. The stem requires elastic subsidence to develop the interface compression to balance the force but also its subsidence is resisted by its curvature. In this respect, the term press-fitted is a contradiction.
The dependence of the load-transfer mechanism on the interface bonding characteristics, which overshadow the effects of stem rigidity, was also noted earlier in analytic and experimental studies. ${ }^{7,24}$ The load transfer mechanism of a press-fit stem depends very much on the stem shape as a geometric entity, whereas for cemented and ingrown stems the shape as a mechanical entity, in terms of rigidity, is more important. A different stem contour can create quite different stress patterns around press-fit stems, as found when comparing the Osteonics to the Zweymüller designs (AlloPro, Baar, Switzerland) ${ }^{16}$ The same argument can be used for the difference between two-dimensional and three-dimensional finite-element models. Stress effects found for the press-fit stem case indicate that two-dimensional finite-element models will be much less accurate in describing the true stress patterns than those for bonded interfaces, particularly when these stems are also tapered in the sagittal plane or structured at their anterior or posterior surfaces.

In the present study, the time factor was not considered. In fact, each coated stem is made as a press-fitted prosthesis, albeit with much higher coefficients of friction. In time, all configurations experience gradual changes in bone structure and mass, affecting bone mechanical properties and interface conditions. The present results particularly emphasize the importance of interface bonding. The load-transfer mechanism in cemented stem configurations change considerably when bone-cement loosening and fibrous-tissue interposition occur. ${ }^{31}$ For the noncemented stems, much depends on fit, stem-surface roughness, and opportunities for bony ingrowth or osseous integration. Because of the inherent surgical variations in bone preparation, it is likely that the postoperative biomechanical behavior of noncemented stems is less predictable than cemented ones. Even if a reasonably uniform fit is obtained over the whole stem region, the noncemented stem (either press-fit or coated) may sink into the diaphysis to be contained there by the relatively 
stiff cortical bone shell. If proximal ingrowth can occur fast, if only in a small region, this would be prevented. If the stem is smooth, with no opportunities for ingrowth, this scenario seems inevitable.

Taking into account the simplicity of the finite-element models used, and the schematic character of the approach, particularly bearing in mind the inherent weakness of the two-dimensional model for the press-fitted prosthesis, the following general conclusions can be made. (1) Considering the four structural aspects governing load transfer in femoral THA, i.e., loading characteristics, geometry, material properties, and interface bonding characteristics, interface bonding is by far the most influential one. (2) The stress patterns in bonded stem configurations, either cemented, fully ingrown, or partly ingrown, are determined predominantly by the bending effects of the hip-joint force. Conversely, when the stem is unbonded, the axial force component has more influence. (3). The stress patterns in cemented and noncemented but bonded configurations are qualitatively similar. Differences in stress values occur because of the bulkiness or rigidity of the stem. The noncemented stems create more cortical stress shielding, more distal stress transfer, and less proximal stress transfer. (4) Considering cement strength, cement-bone interface strength, bonding strength of bony ingrowth or osseous integration, and potential effects of cortical stress shielding on bone resorption, CoCrMo material is preferred for cemented stems and titanium for noncemented stems. (5) Load transfer in smooth press-fit stems depends predominantly on the stem shape as a geometric entity and much less on its rigidity. This also emphasizes the need for three-dimensional finite-element models to determine their precise mechanical behavior. More research is needed to document the interrelationships between shape, fit, and surface roughness of press-fitted stems. (6) Partly coated stems have more potential for creating uniform stress transfer over the full length of the stem than fully coated ones. (7) All stems create severe stress shielding in the calcar region. All bonded stems also create stress shielding farther down the cortex. The amount of stress shielding reduces from fully ingrown, to partly ingrown, to cemented stems.

\section{ACKNOWLEDGMENT}

The author thanks R. v.d. Venne. M.S., for carrying out the finite-element method calculations.

\section{REFERENCES}

1. Ahmed. A. M.. Raab, S.. and Miller. J. E.: Metal-cement interface strength in cemented stem fixation. J. Orthop. Res. 2:105, 1984.

2. Brown. T. D.. Pedersen. D. R., Radin. E. L., and Rose. R. M.: Global mechanical consequences of reduced cement/bone coupling rigidity in proximal femoral arthroplasty: A three dimensional finite element analysis. J. Biomech. 21:115, 1988.

3. Cook. S. D.. Walsh. K. A.. and Haddad. R. J.. Jr.: Interface mechanics and bone growth into porous Co-Cr-Mo alloy implants. Clin. Orthop. 193:271, 1985.

4. Crowninshield. R. D.. Brand, R. A.. Johnston, R. C., and Milroy. J. C.: An analysis of femoral component stem design in total hip arthroplasty. $J$. Borie Jornt Surg. 62A:68, 1980

5. Crowninshield. R. D. Brand, R. A.. Johnston, R.C., and Milroy, J. C.: The effect of femoral stem crosssectional geometry on cement stresses in total hip reconstruction. Clin. Orthop. 146:71, 1980.

6. Crowninshield, R. D., Johnston, R. C., and Andrews, J. G.: A biomechanical investigation of the human hip. J. Biomech. 11:75, 1978.

7. Diegel. P. D.. Daniels. A.U.. and Dunn, H. K.: Initial effect of collanless stem stiffness on femoral bone strain. J. Arthroplasty 4:173, 1989.

8. Draenert. K.: Forschung und Fortbildung in der Chirurgie des Bewegungsapparates 2. Munich, Art and Science 1988.

9. Engh. C. A., and Bobyn. J. D.: The influence of stem size and extent of porous coating on femoral bone resorption after primary cementless hip arthroplasty. Clin. Orthop. 231:7, 1988.

10. Engh. C. A., Bobyn, J. D., and Glossman, A. H.: Porous-coated hip replacement: The factors governing bone ingrowth, stress shielding, and clinical results. J. Bone Joint Surg. 69B:45, 1987.

11. Finlay, J. B., Rorabeck, C. H., Bourne, R. B., and Tew. W. M.: In vitro analysis of proximal femoral strains using PCA femoral implants and a hip-abductor muscle simulator. J. Arthroplasty 4:335, 1989.

12. Geesink, R. G. T.; Hydroxyl-apatite coated hip implants. Dissertation. University of Limburg, Maastricht, The Netherlands, 1988.

13. Huiskes, R.: Some fundamental aspects of humanjoint replacement. Acta Orthop. Scand. 51 [Suppl. 185]:1. 1980.

14. Huiskes. R.: Design, fixation, and stress analysis of 
permanent orthopedic implants: The hip joint. In Ducheyne, P., and Hastings. G. (eds.): Functional Behavior of Biomaterials, vol. 2: Applications. Boca Raton, Florida, CRC Press, 1984, p. 121.

15. Huiskes. R.: Stress patterns, failure modes and bone remodeling. In Fitzgerald, R.. Jr. (ed.): Non-Cemented Total Hip Arthroplasty. New York, Raven Press. 1988, p. 283.

16. Huiskes. R., and Chao, E. Y. S.: A survey of finite element methods in orthopaedic biomechanics. J. Biomech. 16:385. 1983.

1\%. Huiskes, R.. Weinans, H., and Dalstra, M.: Adaptive bone remodeling and biomechanical design considerations for noncemented total hip arthroplasty. Orthopedics 12:1255, 1989

18. Huiskes, R., Weinans, H., Grootenboer. H. J., Dalstra, M.. Fudala, B.. and Slooff, T. J.: Adaptive bone-remodeling theory applied to prosthetic-design analysis. J. Biomech. 20:1135. 1987.

19. Huiskes. R., Weinans, H. Sumner. D. R.. Fudala. B., Turner, T. M., Grootenboer, H. J., and Galante. J. O.: Stress-shielding. stress-bypassing and bone resorption around "press-fit" and bone ingrowth THA. ORS 35th Annual Meeting. Las Vegas. Nevada. Feb. 6-9. 1989.

20. Johnston, R. C.: The case for cemented hips. Iowa Orthop. J. 6:60, 1987.

21. Krause. W. R.. Krug. W.. and Miller. J.: Strength of the cement-bone interface. Clin. Orthop. 163:290. 198 ?.

22. Lewis. J. L., Askew, M. J.. Wixson. R. L.. Kramer. G. M.. and Tarr. R. R.: The influence of prosthetic stem stiffness and of calcar collar on stresses in the proximal end of the femur with a cemented femoral component. J. Bone Joint Surg. 66A:280, 1984.

23. Noble. P. C.. Alexander. J. W. Lindahl. L. J.. Yew D. T.. Granberry. W. M.. and Tullos. H. S.: The anatomic basis of femoral component design. Clin. Orthop. 235:148, 1988.

24. Rohlmann, A., Cheal, J., Hayes, W. C. and Bergmann. G.: A nonlinear finite element analysis of interface condition in porous coated hip endoprostheses. J. Biomech. 21:201, 1988.

25. Rohlmann, A., Moessner, U., Bergmann, G., and Koelbel, R.: Finite element analysis and experimental investigation in a femur with hip endoprosthesis. J. Biomech. 16:727, 1983.

26. Rohlmann. A.. Moessner, U.. Bergmann, G., and Koelbel, R.: Effects of stem design and material properties on stresses in hip endoprostheses. J. Biomed. Eng. 9:77, 1987.

27. Stauffer, R. N.: Ten-year follow-up study of total hip replacement. J. Bone Joint Surg. 64A:983, 1982.

28. Turner, T. M., Sumner, D. R., Urban, R. M., Rivero. D. P., and Galante, J. O.: A comparative study of porous coatings in a weight-bearing total hiparthroplasty model. J. Bone Joint Surg. 68A:1396. 1986.

29. Verdonschot, N.. and Huiskes, R.: FEM analyses of hip prostheses: Validity of the 2-D side-plate model and the effects of torsion. European Society of Biomechanics 7 th Annual Meeting. Arhus, Denmark. July 8-11, 1990.

30. Walker, P. S.. Schneeweis, D., Murphy, S., and Nelson. P.: Strains and micromotions of press-fit femoral stem prostheses. J. Biomech. 20:693, 1987.

31. Weinans, H., Huiskes, R., and Grootenboer, H. J.: Mechanical consequences of a fibrous membrane around femoral hip prostheses. J. Biomech. 1990. (In press.)

32. Zhou, X. M.. Robertson, D. D.. and Walker, P. S.: Femoral strain patterns with press-fit THR:-Photo-elastic analysis. ORS 34th Annual Meeting, Atlanta, Georgia. Feb. 1-4. 1988. 